\title{
Transplant-associated thrombotic microangiopathy and acquired nephrotic syndrome: A case report in a 35-month child.
}

Tascon A. Jose-Andres ( $\sim$ jata0228@hotmail.com )

Antioquia University https://orcid.org/0000-0001-6647-2842

Catalina Velez Echeverri

Antioquia University https://orcid.org/0000-0002-2904-3392

Carolina-Lucia Ochoa Garcia

Pablo Tobon Uribe Hospital https://orcid.org/0000-0003-3706-8159

Arias. Luis $\mathbf{F}$

Antioquia University

Vanegas R. Juan Jose

Antioquia University https://orcid.org/0000-0002-0734-0986

\section{Case Report}

Keywords: Thrombotic Microangiopathy, Atypical Hemolytic Uremic Syndrome, Acute Kidney Injury, Nephrotic Syndrome, Transplantation

Posted Date: February 2nd, 2022

DOI: https://doi.org/10.21203/rs.3.rs-1312682/v1

License: (c) (1) This work is licensed under a Creative Commons Attribution 4.0 International License. Read Full License 


\section{Abstract \\ Background}

Transplant-associated thrombotic microangiopathy (TA-TMA) presents with thrombocytopenia, nonimmune hemolytic anemia, peripheral blood schistocytes and end-organ damage to the kidney. TATMA is associated with a significant increased morbidity and mortality, especially when treatment is not initiated early. We present a pediatric clinical case of a 35-month child with history of hepatic transplantation, who develop the clinical spectrum of TA-TMA and acquired nephrotic syndrome.

\section{Case presentation:}

A 35-month-old boy with history of hepatic transplantation secondary of atresia of biliary ducts who received immunosuppressive therapy with tacrolimus, mycophenolate and steroids. He presents with 4 days of fever, vomiting, and non-dysenteric diarrhea. He received an initial course of antibiotics without response. After 3 days he continues with fever, low urine output and edema. Vital signs showed high blood pressure and physical exam revealed facial and extremity edema. Laboratory results showed proteinuria and hematuria, low albumin, and high triglycerides. All possible etiologies of nephrotic syndrome were ruled out. Kidney biopsy showed TMA changes. Plasma exchange and Eculizumab were started with clinical improvement.

\section{Discussion}

TMA can be classified as a primary or secondary. Primary TMA can be hereditary or acquired. Acquired TMA included the ones triggered by medications. This clinical syndrome has been described as an endothelial dysfunction secondary of an immune reaction over the endothelium and/or a direct cytotoxic effect over endothelium and platelets. In this case report the use of calcineurin inhibitors (tacrolimus) one of the most common drugs associated with TMA was one the triggers factors. Recent publications have described how these patients that are exposed to any triggers has also a genetically predisposing to develop TMA. There is a lack in diagnostics and prognostic markers. The earliest treatment is stared, the better prognosis and outcomes.

\section{Background}

TMA syndrome is characterized by the presence of microangiopathic hemolytic anemia, thrombocytopenia, and end organ damage that could include the kidney. It is described as an endothelial injury in arterioles and capillaries, which generates a pro thrombotic effect with increased lactic acid dehydrogenase (LDH) and decreased haptoglobin (1)(2). The etiologies of TMA syndrome are diverse and range from hereditary, acquired and secondary to the use of medications, some infections, malignancy and autoimmune diseases (3). TMA requires a high index of suspicion in. order to obtain an 
early diagnosis and early initiation of therapy. It is associated with a bad prognosis, high morbidity, and mortality. The development of new therapies has improved the prognosis of these conditions. We present a pediatric case of TMA with multiple trigger factors including medications (tacrolimus) and Epstein-Barr virus (EBV) infection in association with nephrotic syndrome.

\section{Case Presentation}

A 35-month-old Colombian boy with past medical history of extrahepatic biliary duct atresia with hepatic transplantation from live related donor at 19 months of life (without hepatoportoenterostomy/ Kasai), hepatic insufficiency and portal hypertension. He received treatment with prednisolone, tacrolimus, mycophenolate and valganciclovir due to a positive Cytomegalovirus (CMV) viral load (1876 viral copies $/ \mathrm{ml}$ ). He presented to the emergency department in his community hospital with 4 days of fever, vomiting, and diarrhea. He was sent home on antibiotics (Trimethoprim/Sulfamethoxazole) for a presumed diagnosis of bacterial gastroenteritis. Three days later he is still febrile, and the parents started to notice decreased urine output associated with generalized edema. Physical examination at this point was positive for facial and extremity edema, high blood pressure for age and height $(95+12 \mathrm{mmHg}$ percentile). Initial laboratories (Table 1) revealed proteinuria and hematuria, associated with low albumin and high triglycerides as well as elevated creatinine. He was diagnosed with nephrotic syndrome, acute kidney injury (AKI) KDIGO I and normocytic anemia. 
Table 1

Laboratories results.

\begin{tabular}{|lll|}
\hline Laboratory & Result & Reference range \\
\hline Hemoglobin & $7.7 \mathrm{~g} / \mathrm{dL}$ & $11.5-16.1 \mathrm{~g} / \mathrm{dL}$ \\
\hline Platelets & $161.000 / \mathrm{mm}^{3}$ & $150.000-350.000 / \mathrm{mm}^{3}$ \\
\hline Schistocytes & Positive & Negative \\
\hline Reactive C Protein (RCP) & $4.25 \mathrm{mg} / \mathrm{dL}$ & $<0.04 \mathrm{mg} / \mathrm{dL}$ \\
\hline LDH & $939 \mathrm{U} / \mathrm{l}$ & $143-290 \mathrm{U} / \mathrm{I}$ \\
\hline Direct Coombs & Negative & Negative \\
\hline Ferritin & $1110 \mathrm{mcg} / \mathrm{L}$ & $10-120 \mathrm{mcg} / \mathrm{L}$ \\
\hline Albumin & $1.8 \mathrm{~g} / \mathrm{dL}$ & $3.6-5.2 \mathrm{~g} / \mathrm{dL}$ \\
\hline Creatinine & $0.76 \mathrm{mg} / \mathrm{dL}$ & $0.45-0.5 \mathrm{mg} / \mathrm{dL}$ \\
\hline Triglycerides & $198 \mathrm{mg} / \mathrm{dL}$ & $<150 \mathrm{mg} / \mathrm{dL}$ \\
\hline Complement C4 & $4 \mathrm{mg} / \mathrm{dL}$ & $7-40 \mathrm{mg} / \mathrm{dL}$ \\
\hline Urine: & $150 \mathrm{mg} / \mathrm{dL}$ & Negative \\
Uranalysis: Proteins and Erythrocytes & $6-10 \mathrm{por}$ CAP & Negatives \\
\hline 24 hours protein & $4 \mathrm{grams}$ & $0-4 \mathrm{mg} / \mathrm{m}^{2} / \mathrm{hora}$ \\
\hline EBV Viral load & $53.200 \mathrm{copies}$ & Negative \\
\hline
\end{tabular}

*Reference: Soghier L. Reference Range Values for Pediatric Care. AAP (2014). elSBN: 978-1-58110-854-5.

Due to the history of immunosuppression and CMV infection, infectious etiologies of the nephrotic syndrome were ruled out. Repeat CMV viral load was negative, HIV, Adenovirus, Rotavirus and Influenza A and B were negative. Epstein-Barr virus (EBV) viral load was positive with 53.000 viral copies. Blood and urine cultures were negative.

During his hospital course he remained with high blood pressure, non-immune hemolytic anemia with schistocytes in the peripheral blood smear, increased LDH and platelets dropped more that $25 \%$. The diagnosis of thrombotic microangiopathy was made, in association with nephrotic syndrome. Differential diagnosis included: post-transplant lymphoproliferative disorder (PTLD) related with a positive EBV viral load, increased LDH and history of tacrolimus; Hemophagocytic syndrome secondary to EBV infection and history of fever, anemia, leucopenia, thrombocytopenia, high triglycerides, high LDH and increased ferritin. However, bone marrow studies did not suggest either PTLD or hemophagocytic syndrome. Additional laboratories showed ADAMTS13 of $45 \%$ ruling out TTP, decreased C4 with normal C3, and normal antinuclear antibodies (ANA) 1:40 with cytoplasmic pattern, extractable nuclear antigen (ENA), 
Antineutrophil Cytoplasmic Antibodies (ANCA) and Antiglomerular basement membrane antibodies. Hepatic function was normal during the whole course.

Renal biopsy (Figure 1) showed endothelial edema, double contours with negative immunofluorescence and focal and segmental sclerotic glomerular lesions. Pathology concluded that these changes were compatible with TMA. Patient was started on plasma exchange and Eculizumab. Clinical and laboratory improvement was achieved, and the patient was discharged on oral steroids and close follow up as an outpatient.

\section{Discusion And Conclusion}

TMA are classified as primary or secondary. Primary TMAs are divided in hereditary or acquired. Hereditary TMA include those who are mediated by complement mutations (denominated as atypical hemolytic syndrome, aHUS) which generate a non-controlled activation of the alternative pathway of the complement with $\mathrm{C} 3$ fraction consumption. For this case, a hereditary TMA was considered as a diagnosis even though the $\mathrm{C} 3$ fraction was normal, because this does not exclude the diagnosis and the final diagnosis should be made by molecular studies that identify the specific mutation. (2). From the hereditary TMA, thrombotic thrombocytopenic purpura was ruled out due to the normal result of ADAMTS $13(>10 \%)$. For acquired etiologies of TMA in which there are antibodies that directly target and inhibit ADMATS13 or $\mathrm{H}$ factor of the complement, they present with decreased $\mathrm{C} 4$ as the current case, however, there was no other clinical or laboratories findings to make this diagnosis.

Another acquired etiologies of TMA are those who are triggered by medications that could generate an immune reaction and/or cytotoxic reaction to the endothelium, specifically to the renal endothelium with an increase in platelet function and aggregation (1)(4). In this case, one of the risk factors was the previous use of Tacrolimus, a calcineurin inhibitor. These groups of medications and the tacrolimus itself has been implicated as one of the most common medications that triggers these reactions (1). The clinical presentation of TMA triggered by medications can be as early as 21 days after initial use (early presentation) and up to weeks or months (late presentation). Renal compromise is characterized by AKI, C3 and C4 can be normal or decreased, with normal ADAMTS13. In recent literature the term transplantassociated thrombotic microangiopathy (TA-TMA) has been described specially in stem cells transplant patients. Some academic groups have postulated some diagnostic criteria, which are used to guide the diagnostic process (5). We considered under the scope of these criteria that the patient described in this case fulfill most of them as there is presence of schistocytes, high LDH, thrombocytopenia, non-immune hemolytic anemia, renal compromise with normal coagulation laboratories.

Nwaba $A$ et al described 4 patients after liver transplantation who developed TMA due to the use of tacrolimus, they were all negative for CMV, Parvovirus B19, BK virus, HIV and EBV (3). Devadoss CW et al. Described a case of TA-TMA in a renal transplanted patient associated with tacrolimus, with negative infections serologies as well (6). For this case, the patients present with a positive EBV viral load which we consider could play a role as a trigger factor in the whole clinical scenario. Infectious etiologies 
described for TMA include bacterial, parasites, and viruses. Viruses have been related with an endothelium damage that can precipitate the TMA. For the specific case of EBV is less common as an etiology because the glomerular endothelial cells lack CD21 antigen, which is the main receptor for EVB (7).

Among the diagnostic possibilities, secondary hemophagocytic syndrome was considered, especially with the present of positive EVB positive viral load (8). Ramos-Casals et al. described possible trigger factors for the development of this syndrome and some of them were present in the current case: positive EVB virus load and history of organ solid transplantation. The renal compromise in this syndrome is described with AKI (88\%) and nephrotic syndrome (38\%) with focal and segmental sclerosis changes in the biopsy (9). Bone marrow biopsy did not suggest the presence of hemophagocytic syndrome and PTLD as there was no hemophagocytosis or a monoclonal B cell population. There is a TAM that is described as the novo in solid organ transplantations, despite this patient was a solid organ recipient, the incidence of this diagnosis is between 0 to $3.9 \%$ in liver transplantation and the time frame in which it can present is between 2 to 8 weeks, which made this diagnosis less likely.

Nephrotic syndrome is defined as the presence of proteinuria ( $>2 \mathrm{~g} /$ day or $>40 \mathrm{mg} / \mathrm{m}^{2} /$ hour), associated with low albumin and high triglycerides, with clinical edema. Infectious etiologies of this syndrome includes CMV, Parvovirus B19, HIV, hepatitis B, C, A and EBV (10). Other etiologies are secondary to medications, including tacrolimus (11).

After taking all of these into account, it was considered that the development of nephrotic syndrome in this patient was multifactorial, etiologies include the use of tacrolimus and a possible genetic susceptibility that eventually developed a TA-TMA, associated with risk factors as being a solid organ transplanted patient and has a EVB positive viral load as a major trigger factor either for TMA and nephrotic syndrome (2)(5)(7).

\section{References}

1. George JN, Nester CM. Syndromes of Thrombotic Microangiopathy. N Engl J Med. 14 de agosto de 2014;371(7):654-66.

2. Brocklebank V, Wood KM, Kavanagh D. Thrombotic Microangiopathy and the Kidney. Clin J Am Soc Nephrol. 7 de febrero de 2018;13(2):300-17.

3. Nwaba A, MacQuillan G, Adams LA, Garas G, Delriviere L, Augustson B, et al. Tacrolimus-induced thrombotic microangiopathy in orthotopic liver transplant patients: case series of four patients. Intern Med J. marzo de 2013;43(3):328-33.

4. Al-Nouri ZL, Reese JA, Terrell DR, Vesely SK, George JN. Drug-induced thrombotic microangiopathy: a systematic review of published reports. Blood. 22 de enero de 2015;125(4):616-8.

5. Gavriilaki E, Sakellari I, Anagnostopoulos A, Brodsky RA. Transplant-associated thrombotic microangiopathy: opening Pandora's box. Bone Marrow Transplant. octubre de 2017;52(10):1355- 
60.

6. Devadoss CW. Tacrolimus Associated Localized Thrombotic Microangiopathy Developing in Early Stage after Renal Transplantation. J Clin Diagn Res [Internet]. 2012 [citado 31 de mayo de 2020]; Disponible en: http://www.jcdr.net/article_fulltext.asp?issn=0973709x\&year $=2012 \&$ volume $=6 \&$ issue $=10$ \&page $=1786 \&$ issn $=0973-709 x \&$ id $=2614$

7. Lopes da Silva R. Viral-associated thrombotic microangiopathies. Hematol Oncol Stem Cell Ther. abril de 2011;4(2):51-9.

8. Cupit-Link MC, Kohorst MA, Tran CL, Bleesing JJ, Galardy PJ, Boyce TG. Suspected thrombotic microangiopathy in a child with Epstein-Barr virus-induced hemophagocytic lymphohistiocytosis. Pediatr Blood Cancer. junio de 2018;65(6):e27002.

9. Ramos-Casals M, Brito-Zerón P, López-Guillermo A, Khamashta MA, Bosch X. Adult haemophagocytic syndrome. The Lancet. abril de 2014;383(9927):1503-16.

10. Wang C, Greenbaum LA. Nephrotic Syndrome. Pediatr Clin North Am. febrero de 2019;66(1):73-85. 11. D’Agati VD. Focal Segmental Glomerulosclerosis. N Engl J Med. 2011;14.

\section{Declarations}

Statement on participant consent: for the current case report, the legal guardian has consented to participate in the publication of this case and for an eventually publication, this include clinical information and images (biopsy). Statement for potential competing interests: the authors declare no competing interests.

\section{Figures}




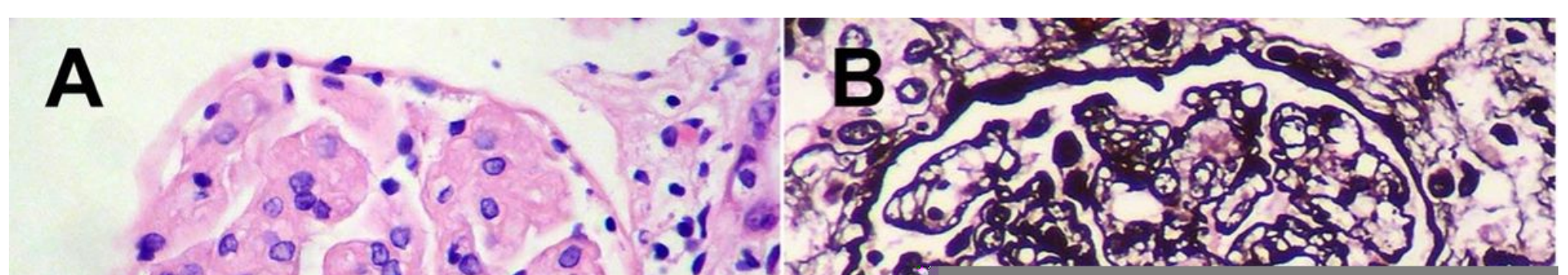

\section{Figure 1}

A: glomerulus with a compacted appearance, with thickened capillary walls and a marked decrease in its lumens, endothelial edema, and a bloodless appearance (hematoxylin-eosin, X400). B: Silver staining reveals some double contours of the capillary wall, with irregularly thickened (methenamine-silver, X400). C: In this glomerulus, two segments of sclerosis are evident, marked by collagen proliferation, positive with silver staining, and loss of capillary lumens (silver-methenamine, X400). D: ultrastructural study: basement membrane of a glomerular capillary which is thickened and irregular; there is loss of podocyte processes on almost the entire surface of this capillary; endothelium with loss of fenestrations and edema (transmission electron microscopy, original magnification, $\mathrm{X} 2,500$ ). 